\title{
Aharonov-Bohm Effect and the Supersymmetry of Identical Anyons
}

\author{
V. Jakubský
}

\begin{abstract}
We briefly review the relation between the Aharonov-Bohm effect and the dynamical realization of anyons. We show how the particular symmetries of the Aharonov-Bohm model give rise to the (nonlinear) supersymmetry of the two-body system of identical anyons.
\end{abstract}

Keywords: nonlinear supersymmetry, Aharonov-Bohm effect, Anyons.

\section{Aharonov-Bohm effect}

More than fifty years ago, Aharonov and Bohm argued in their seminal paper [1] that the fundamental quantity in a description of the quantum system is the electromagnetic potential and not the electromagnetic field. They proposed an experiment in which two beams of electrons are guided around a thin solenoid that is shielded completely from the electrons. Despite the absence of the magnetic field outside the solenoid, the wave functions are affected by the non-vanishing electromagnetic potential and acquire an additional phase-factor which is manifested in the altered interference of the beams. The so-called Aharonov-Bohm (AB) effect has been observed experimentally [2] and has found its application in numerous fields of physics. In the present article, we will review its relation to anyons, twodimensional particles of exotic statistics. We will present the recent results on the nonlocal symmetries of the $\mathrm{AB}$ system and their relation to the supersymmetry of two-body anyon models.

Let us consider a spin-1/2 particle which is moving in a plane. The plane is punctured perpendicularly in the origin by an infinitely thin solenoid. The solenoid is impenetrable for the particle. Hence, the origin is effectively removed from the space where the particle lives. The Pauli Hamiltonian of the system acquires the following simple form ${ }^{1}$

$$
\mathcal{H}=\frac{1}{2 m} \sum_{j=1,2} \mathcal{P}_{j}^{2}-\frac{e \hbar}{2 m c} B_{3} \sigma_{3},
$$

where $\mathcal{P}_{j}=-i \hbar \partial_{j}-\frac{e}{c} A_{j}, B_{3}=\partial_{1} A_{2}-\partial_{2} A_{1}$. The non-vanishing electromagnetic potential in the symmetric gauge reads

$$
\vec{A}=\frac{\Phi}{2 \pi}\left(-\frac{x_{2}}{x_{1}^{2}+x_{2}^{2}}, \frac{x_{1}}{x_{1}^{2}+x_{2}^{2}}, 0\right)=
$$

\footnotetext{
${ }^{1}$ We set $m=1 / 2, \hbar=c=-e=1$ from now on.
}

${ }^{2}$ In fact, the Dirac delta term in the Hamiltonian is quite formal. It can be omitted when the domain of $\mathcal{H}_{\alpha}$ is specified correctly.

$$
\frac{\Phi}{2 \pi r}(-\sin \varphi, \cos \varphi, 0)
$$

where $x_{1}=r \cos \varphi, x_{2}=r \sin \varphi,-\pi<\varphi \leq \pi$, and $\Phi$ is the flux of the singular magnetic field, $B_{3}=\Phi \delta^{2}\left(x_{1}, x_{2}\right)$. As we will work mostly in polar coordinates, let us present the explicit form of the Hamiltonian in this coordinate system

$$
\begin{aligned}
\mathcal{H}_{\alpha} & =-\partial_{r}^{2}-\frac{1}{r} \partial_{r}+\frac{1}{r^{2}}\left(-i \partial_{\varphi}+\alpha\right)^{2}+\alpha \frac{1}{r} \delta(r) \sigma_{3}, \\
\alpha & =\frac{1}{2 \pi} \Phi
\end{aligned}
$$

Here we used the identity $\delta^{2}\left(x_{1}, x_{2}\right)=\frac{1}{\pi r} \delta(r)$ for the two dimensional Dirac delta function ${ }^{2}$.

To specify the system uniquely, we have to determine the domain of the Hamiltonian. We require the operator (1.3) to act on $2 \pi$-periodic functions $\Psi(r, \varphi)$, i.e. $\Psi(r, \varphi+2 \pi)=\Psi(r, \varphi)$. Using the expansion in partial waves, we can write

$$
\Psi(r, \varphi)=\sum_{j} e^{i j \varphi} f_{j}(r)
$$

The functions $f_{j}(r)$ should be locally squareintegrable (i.e. $f_{j}(r)$ should be square integrable on any finite interval). The partial waves $f_{j}(r)$ are regular at the origin up to the exception specified by the following boundary condition

$$
\lim _{r \rightarrow 0^{+}} \Psi \sim\left(\begin{array}{l}
\left(1+e^{i \gamma}\right) 2^{-\alpha} \Gamma(1-\alpha) r^{-1+\alpha} e^{-i \varphi} \\
\left(1-e^{i \gamma}\right) 2^{-1+\alpha} \Gamma(\alpha) r^{-\alpha}
\end{array}\right)
$$

where parameter $\gamma$ can acquire two discrete values 0 and $\pi$. The boundary condition (1.5) is related to the self-adjoint extensions of the Hamiltonian. Let us note that the boundary condition (1.5) just fixes two self-adjoint extensions (one for $\gamma=0$, the second one for $\gamma=\pi$ ) of the formal operator $\mathcal{H}_{\alpha}$ that are 
compatible with the existence of $N=2$ supersymmetry, see [3]. To keep our presentation as simple as possible, we fix from now on

$$
\gamma=0
$$

We modify the actual notation to indicate the domain of the Hamiltonian, i.e. we will write $\mathcal{H}_{\alpha} \rightarrow \mathcal{H}_{\alpha}^{0}$. For readers who are eager for a more extensive analysis of the problem we recommend [3] for reference.

The Hamiltonian $\mathcal{H}_{\alpha}^{0}$ commutes with the angular momentum operator $J=-i \partial_{\varphi}+\alpha$ and the spin projection $s_{3}=\frac{1}{2} \sigma_{3}$. Hence, one can find the vectors $|E, l, s\rangle$ such that

$$
\begin{aligned}
\mathcal{H}_{\alpha}^{0}|E, l, s\rangle & =E|E, l, s\rangle \\
J|E, l, s\rangle & =(l+\alpha)|E, l, s\rangle \\
s_{3}|E, l, s\rangle & =s|E, l, s\rangle .
\end{aligned}
$$

We define the following two additional integrals of motion

$$
\begin{aligned}
Q & =\sigma_{1} \mathcal{P}_{1}+\sigma_{2} \mathcal{P}_{2}=q_{+} \sigma_{+}+q_{-} \sigma_{-}, \\
q_{ \pm} & =-i e^{\mp i \varphi}\left(\partial_{r} \pm \frac{1}{r}\left(-i \partial_{\varphi}+\alpha\right)\right) \\
\sigma_{ \pm} & =\frac{1}{2}\left(\sigma_{1} \pm i \sigma_{2}\right)
\end{aligned}
$$

and

$$
\begin{aligned}
\tilde{Q} & =\mathcal{P}_{1} \mathbf{1}+i R \sigma_{3} \mathcal{P}_{2}, \\
R r R & =r, \\
R \varphi R & =\varphi+\pi,
\end{aligned}
$$

where $\mathbf{1}$ is a unit matrix and

$$
\begin{aligned}
\mathcal{P}_{1}+i R \mathcal{P}_{2} & =q_{+} \Pi_{+}+q_{-} \Pi_{-}, \\
\Pi_{ \pm} & =\frac{1}{2}(1 \pm R) .
\end{aligned}
$$

We can make a qualitative analysis of how these operators act on the wave functions $|E, l, s\rangle$ just by observing their explicit form. For instance, we have

$$
\begin{aligned}
Q|E, l, 1 / 2\rangle & \sim|E, l+1,-1 / 2\rangle, \\
\tilde{Q}|E, 2 l, s\rangle & \sim|E, 2 l-2 s, s\rangle .
\end{aligned}
$$

Hence, neither $Q$ nor $\tilde{Q}$ commutes with the angular momentum $J$ and the parity $R$. However, the operator $\tilde{Q}$ preserves spin of the wave functions, i.e. $\left[\tilde{Q}, s_{3}\right]=0$.

The operators $Q$ and $\tilde{Q}$ are related by nonlocal unitary transformation, see [4]. In addition, we can define

$$
\mathcal{W}=Q \tilde{Q}=\tilde{Q} Q .
$$

This operator alters both the angular momentum and the spin of the wave functions. The explicit action of $Q, \tilde{Q}$ and $\mathcal{W}$ on the kets $|E, l, s\rangle$ is illustrated in Fig. 1.

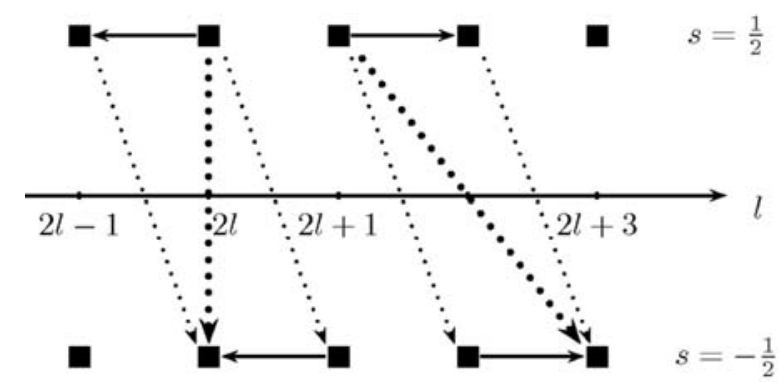

Fig. 1: The action of operator $\mathcal{W}$ (thick dotted arrows) on the states $|E, 2 l, 1 / 2\rangle$ and $|E, 2 l+1,1 / 2\rangle$ as a sequential action of $\tilde{Q}$ (solid arrows) and $Q$ (thin dotted arrows). Black squares represent the eigenstates $|E, l, s\rangle$ with corresponding values of $l$ and $s$

\section{Anyons}

Quantum theory has classified particles into two disjoint families; there are bosons with integer spin and fermions with half-integer spin. The wave functions of indistinguishable bosons or fermions reflect the specific statistical properties of the particles. When we exchange two bosons, the wave function remains the same. When we exchange two fermions, the corresponding wave function changes the sign. The wave functions respect either Bose-Einstein or Fermi-Dirac statistics in this way.

However, when one makes a quantum system be two-dimensional, there emerges an alternative to the classification.

As predicted by Wilczek [5], there can exist exotic particles in two-dimensional space that are called anyons. Anyons interpolate between bosons and fermions in the sense that when we exchange two of them in the system, the associated wave function acquires a multiplicative phase-factor of unit amplitude but distinct from \pm 1 . The prediction of these particles is physically relevant for various condensed matter systems where the dynamics is effectively twodimensional.

Wilczek proposed a simple dynamical realization of anyons with the use of "composite" particles. Let us explain the idea on a simple model of two identical particles [6]. Take either two bosons or fermions. Then, glue each of the particles together with a magnetic vortex, i.e. with infinitely thin solenoids of the same magnetic flux $\alpha$. As a result, we get two identical composite particles. Each particle can "see" just the potential generated by the solenoid of the other particle. The Hamiltonian corresponding to this twobody system has the following form

$$
H_{a n y}=2 \sum_{I=1}^{2}\left(\vec{p}_{I}-\vec{a}_{I}(\vec{r})\right)^{2} .
$$


where $\vec{p}_{I}=-i \partial / \partial \vec{x}_{I}, \vec{r}=\vec{x}_{1}-\vec{x}_{2}$ and the index $I \in\{1,2\}$ labels the individual particles. The potential

$$
a_{1}^{k}(\vec{r})=-a_{2}^{k}(\vec{r})=\frac{1}{2} \alpha \epsilon^{k l} \frac{r^{l}}{\vec{r}^{2}}
$$

encodes the "statistical" interaction of the particles. In this sense, we call $\vec{a}_{I}$ the statistical potential. When we write the Hamiltonian in center-of-the-mass coordinates, the relative motion of the particles is governed by the effective Hamiltonian

$$
H_{r e l}=-\partial_{r}^{2}-\frac{1}{r} \partial_{r}+\frac{1}{r^{2}}\left(-i \partial_{\varphi}+\alpha\right)^{2},
$$

where $r$ is the distance between the particles and $\varphi$ measures their relative angle.

The Hamiltonian (2.3) manifests the relation between the two-body model of identical anyons and the $\mathrm{AB}$ system; formally, it coincides with $\mathcal{H}_{\alpha}^{0}$ up to the irrelevant Dirac delta term. However, its domain of definition is quite different. When anyons (composite particles) are composed of bosons, the wave function has to be invariant under the substitution $\varphi \rightarrow \varphi+\pi$ that corresponds to the exchange of the particles. When anyons are composed of fermions, the wave function has to change the sign after the substitution. Hence, the wave functions are of two types

$$
\begin{aligned}
\psi_{\alpha}(r, \varphi) & =\sum_{l} e^{i l \varphi} f_{\alpha, l}(r), \\
l & \in\left\{\begin{array}{l}
2 \mathbb{Z} \text { for anyons based on bosons }, \\
2 \mathbb{Z}+1 \text { for anyons based on fermions } .
\end{array}\right.
\end{aligned}
$$

We shall explain how the considered model explains anyons as the interpolation between bosons and fermions. We can transform the system by a unitary mapping $U=e^{i \varphi \alpha}$ and describe alternatively the system of two identical anyons by the Hamiltonian $\tilde{H}_{r e l}=U H_{r e l} U^{-1}=-\partial_{r}^{2}-1 / r \partial_{r}+$ $\left(-i \partial_{\varphi}\right)^{2} / r^{2}$. It coincides with the energy operator of the free motion. The simplicity of the Hamiltonian is traded for the additional gauge factor that appears in the wave functions, $\tilde{\psi}_{\alpha}(r, \varphi)=U \psi_{\alpha}(r, \varphi)=$ $e^{i \varphi \alpha} \sum_{l} e^{i l \varphi} f_{\alpha, l}(r)$. The wave functions $\tilde{\psi}_{\alpha}(r, \varphi)$ acquire the phase $e^{i \pi \alpha}$ after the substitution $\varphi \rightarrow \varphi+\pi$ and, hence, interpolate between the values corresponding to Bose-Einstein and Fermi-Dirac statistics.

We are ready to reconsider the $\mathrm{AB}$ system and its symmetries in the framework of identical anyons. The Hamiltonian $\mathcal{H}_{\alpha}^{0}$ can be rewritten as a direct sum with subsystems of fixed value of $\operatorname{spin} s_{3}$ and parity $R$. It is convenient to use the notation that reflects the decomposition of the wave functions into these subspaces, $\tilde{\Psi}=\left(\Psi \Sigma_{+} \Pi_{+}, \Psi \Sigma_{+} \Pi_{-}, \Psi \Sigma_{-} \Pi_{+}, \Psi \Sigma_{-} \Pi_{-}\right)^{T}$ where $\Sigma_{ \pm}=\frac{1}{2}\left(1 \pm \sigma_{3}\right)$ and $\Pi_{ \pm}=\frac{1}{2}(1 \pm R)$. In this formalism, the Hamiltonian reads

$$
\begin{aligned}
\mathcal{H}_{\alpha}^{\gamma=0} & =\operatorname{diag}\left(H_{\alpha,+}^{0}, H_{\alpha,-}^{0}, H_{\alpha,+}^{A B}, H_{\alpha,-}^{A B}\right) \\
H_{\alpha, \pm}^{0} & =\mathcal{H}_{\alpha}^{0} \Sigma_{+} \Pi_{ \pm} \\
H_{\alpha, \pm}^{A B} & =\mathcal{H}_{\alpha}^{0} \Sigma_{-} \Pi_{ \pm}
\end{aligned}
$$

Let us make a few comments on the elements of (2.5). Consider $H_{\alpha,+}^{A B}$ in more detail first. It acts on the wave functions that are periodic in $\pi$. Hence, it can be interpreted as the Hamiltonian of the relative motion of two identical anyons based on bosons. Its wave functions are regular at $r \rightarrow 0$, which can be interpreted as a consequence of a hard-core interaction between the anyons. It is worth noting that the system represented by $H_{\alpha,+}^{A B}$ coincides with the system represented by $H_{\alpha,+}^{0}$. Indeed, the Hamiltonians coincide not only formally but in their domains as well (there are no singular wave functions in their domains, see (1.5)). Hence, we can write $H_{\alpha,+}^{0}=H_{\alpha,+}^{A B}$.

The operators $H_{\alpha,-}^{A B}$ and $H_{\alpha,-}^{0}$ describe the systems of two identical anyons based on fermions. The operator $H_{\alpha,-}^{A B}$ prescribes hard-core interaction between anyons. By contrast, the system described by $H_{\alpha,-}^{0}$ allows singular wave functions. It can be understood as a consequence of a nontrivial contact interaction between the composite particles.

The integrals of motion $Q, \tilde{Q}$ and $\mathcal{W}$ shall be rewritten in the $4 \times 4$-matrix formalism. They read explicitly

$$
\begin{aligned}
Q & =\left(\begin{array}{cccc}
0 & 0 & 0 & q_{+} \\
0 & 0 & q_{+} & 0 \\
0 & q_{-} & 0 & 0 \\
q_{-} & 0 & 0 & 0
\end{array}\right), \\
\tilde{Q} & =\left(\begin{array}{cccc}
0 & q_{-} & 0 & 0 \\
q_{+} & 0 & 0 & 0 \\
0 & 0 & 0 & q_{+} \\
0 & 0 & q_{-} & 0
\end{array}\right), \\
\mathcal{W} & =\left(\begin{array}{cccc}
0 & 0 & q_{+} q_{-} & 0 \\
0 & 0 & 0 & q_{+}^{2} \\
q_{-} q_{+} & 0 & 0 & 0 \\
0 & q_{-}^{2} & 0 & 0
\end{array}\right),
\end{aligned}
$$

where $q_{ \pm}$was defined in (1.8). Substituting (2.5) and (2.6) into the relations $\left[Q, \mathcal{H}_{\alpha}^{\gamma}\right]=0,\left[\tilde{Q}, \mathcal{H}_{\alpha}^{\gamma}\right]=0$ and $\left[\mathcal{W}, \mathcal{H}_{\alpha}^{\gamma}\right]=0$ we get the following set of independent intertwining relations

$$
\begin{array}{cc}
H_{+}^{0} q_{-}=q_{-} H_{-}^{0}, & q_{+} H_{+}^{0}=H_{-}^{0} q_{+}, \\
H_{+}^{0} q_{+}=q_{+} H_{-}^{A B}, & q_{-} H_{+}^{0}=H_{-}^{A B} q_{-}, \\
H_{-}^{A B} q_{-}^{2}=q_{-}^{2} H_{-}^{0}, & q_{+}^{2} H_{-}^{A B}=H_{-}^{0} q_{-}^{2} .
\end{array}
$$


Let us focus on the first set (2.7). They can be rewritten as

$$
\left[\mathbf{q}_{a}^{(1)}, \mathbf{h}^{(1)}\right]=0,
$$

where we used the operators

$$
\begin{aligned}
\mathbf{h}^{(1)} & =\left(\begin{array}{cc}
H_{+}^{0} & 0 \\
0 & H_{-}^{0}
\end{array}\right), \\
\mathbf{q}_{1}^{(1)} & =\left(\begin{array}{cc}
0 & q_{-} \\
q_{+} & 0
\end{array}\right), \\
\mathbf{q}_{2}^{(1)} & =i\left(\begin{array}{cc}
0 & -q_{-} \\
q_{+} & 0
\end{array}\right) .
\end{aligned}
$$

The operators (2.11) close for $N=2$ supersymmetry ${ }^{3}$. Indeed, they satisfy the commutation relation

$$
\left\{\mathbf{q}_{a}^{(1)}, \mathbf{q}_{b}^{(1)}\right\}=2 \delta_{a, b} \mathbf{h}^{(1)}, \quad a, b=1,2 .
$$

Hence, operator $\mathbf{h}^{(1)}$ can be understood as the superextended Hamiltonian of the two-body anyonic systems. The system represented by $H_{+}^{0}$ is based on bosons (the wave functions are $\pi$-periodic), the other system (represented by $H_{-}^{0}$ ) is based on fermions with nontrivial contact interaction. The supercharges $\mathbf{q}_{a}^{(1)}$ provide the mapping between these two systems. They exchange the bosons with fermions within the composite particles. Besides, they switch on (off) the nontrivial contact interaction between the anyons.

The relations (2.8) can be analyzed in the same vein, giving rise to the $N=2$ supersymmetric system of the pair of two-body anyonic models. For the sake of completeness, we present the corresponding operators and the algebraic relations of the superalgebra

$$
\begin{aligned}
& \mathbf{h}^{(2)}=\left(\begin{array}{cc}
H_{+}^{0} & 0 \\
0 & H_{-}^{A B}
\end{array}\right), \\
& \mathbf{q}_{1}^{(2)}=\left(\begin{array}{cc}
0 & q_{+} \\
q_{-} & 0
\end{array}\right), \\
& \mathbf{q}_{2}^{(2)}=i\left(\begin{array}{cc}
0 & -q_{+} \\
q_{-} & 0
\end{array}\right),
\end{aligned}
$$

$$
\begin{aligned}
{\left[\mathbf{q}_{a}^{(2)}, \mathbf{h}^{(2)}\right] } & =0 \\
\left\{\mathbf{q}_{a}^{(2)}, \mathbf{q}_{b}^{(2)}\right\} & =2 \delta_{a, b} \mathbf{h}^{(2)} \\
a, b & =1,2 .
\end{aligned}
$$

The only difference appears in the contact interaction between the anyons. This time, the hard-core interaction appears in both systems (neither $H_{+}^{0}$ nor $H_{-}^{A B}$ has singular wave functions in its domain).

A qualitatively different situation occurs in the last case (2.9). The intertwining relations define the $N=2$ nonlinear supersymmetry ${ }^{4}$ represented by the operators

$$
\begin{aligned}
& \mathbf{h}^{(3)}=\left(\begin{array}{cc}
H_{-}^{0} & 0 \\
0 & H_{-}^{A B}
\end{array}\right), \\
& \mathbf{q}_{2}^{(3)}=\left(\begin{array}{cc}
0 & q_{+}^{2} \\
q_{-}^{2} & 0
\end{array}\right), \\
& \mathbf{q}_{2}^{(3)}=i\left(\begin{array}{cc}
0 & -q_{+}^{2} \\
q_{-}^{2} & 0
\end{array}\right) .
\end{aligned}
$$

They satisfy the following relations

$$
\begin{aligned}
{\left[\mathbf{q}_{a}^{(3)}, \mathbf{h}^{(3)}\right] } & =0 \\
\left\{\mathbf{q}_{a}^{(3)}, \mathbf{q}_{b}^{(3)}\right\} & =2 \delta_{a b}\left(\mathbf{h}^{(3)}\right)^{2}, \\
a, b & =1,2 .
\end{aligned}
$$

The supercharges $\mathbf{q}^{(3)}$ alter the contact interaction between the anyons (hard-core in $H_{-}^{A B}$ to nontrivial in $H_{-}^{0}$ and vice versa) but do not alter the nature of the composite particles.

\section{Comments}

In this paper, we have utilized the intimate relation between the Aharonov-Bohm model and the dynamical realization of anyons in order to construct three different $N=2$ supersymmetric systems of identical anyons. The origin of the supersymmetry can be attributed to the symmetries $Q, \tilde{Q}$ and $\mathcal{W}$ of the

\footnotetext{
${ }^{3}$ Let us suppose that we have a quantum mechanical system described by a Hamiltonian $H$. There are $N$ additional observables, represented by the operators $Q_{a}, a \in\{1, \ldots, N\}$. It is said that the system has supersymmetry, as long as operators $Q_{a}$ together with the Hamiltonian satisfy the following algebraic relations

$$
\left\{Q_{a}, Q_{b}\right\} \sim H \delta_{a b}
$$

If this is the case, operators $Q_{a}$ are called supercharges. As a direct consequence of (2.12), they satisfy the relations

$$
Q_{j}^{2} \sim H, \quad\left[Q_{j}, H\right]=0
$$

${ }^{4}$ The system has nonlinear supersymmetry when the supercharges $Q_{a}, a \in\{1, \ldots, N\}$ satisfy the generalized anticommutation relation [7]

$$
\left\{Q_{a}, Q_{b}\right\}=\delta_{a b} f(H)
$$

where $f(H)$ is a function of the Hamiltonian $H$. Usually, $f(H)$ is considered to be a higher-order polynomial.
} 
spin $-1 / 2$ particle in the field of a magnetic vortex. Reduction of these operators into the specific subspaces (of the fixed value of spin and parity) gave rise to supersymmetry of the anyon systems. A similar construction was recently employed in the case of the reflectionless Poschl-Teller system [8]. Its supersymmetric structure originated from the geometrical symmetries of a higher-dimensional system living in $A d S_{2}$ space after the reduction to the subspaces with a fixed angular momentum value.

\section{Acknowledgement}

The author was supported by grant LC06002 of the Ministry of Education, Youth and Sports of the Czech Republic.

\section{References}

[1] Aharonov, Y., Bohm, D.: Significance of electromagnetic potentials in the quantum theory, Phys. Rev. 115, 485 (1959).

[2] Endo, J., Kawasaki, T., Matsuda, T., Osakabe, N., Tonomura, A., Yamada, H., Yano, S.: Evidence for Aharonov-Bohm effect with magnetic field completely shielded from electron wave, Phys. Rev. Lett. 56, 792 (1986);

Endo, J., Kawasaki, T., Matsuda, T., Osakabe, N., Tonomura, A., Yamada, H., Yano, S.: Experimental confirmation of Aharonov-Bohm effect using a toroidal magnetic field confined by a superconductor, Phys. Rev. A 34, 815 (1986).

[3] Correa, F., Falomir, H., Jakubský, V., Plyushchay, M. S.: Supersymmetries of the $\operatorname{spin}-1 / 2$ particle in the field of magnetic vortex, and anyons, arXiv:1003.1434 [hep-th];

Correa, F., Falomir, H., Jakubský, V., Plyushchay, M. S.: Hidden superconformal symmetry of spinless Aharonov-Bohm system, J. Phys. A 43, 075202 (2010).

[4] Jakubský, V., Nieto, L. M., Plyushchay, M. S.: The origin of the hidden supersymmetry, arXiv:1004.5489 [hep-th].

[5] Wilczek, F.: Magnetic flux, angular momentum, and statistics, Phys. Rev. Lett. 48, 1144 (1982); Quantum mechanics of fractional spin particles, Phys. Rev. Lett. 49, 957 (1982).

[6] Wilczek, F.: Fractional statistics and anyon superconductivity, World Scientific, Singapore (1990);

Khare, A.: Fractional statistics and quantum theory, World Scientific, Singapore (1997).

[7] Andrianov, A. A., Ioffe, M. V., Spiridonov, V. P.: Higher derivative supersymmetry and the Witten index, Phys. Lett. A 174, 273 (1993), [arXiv:hepth/9303005].

[8] Correa, F., Jakubský, V., Plyushchay, M. S.: Aharonov-Bohm effect on $\mathrm{AdS}_{2}$ and nonlinear supersymmetry of reflectionless Poschl-Teller system, Annals Phys. 324, 1078 (2009), [arXiv:0809.2854 [hep-th]].

Ing. Vít Jakubský, Ph.D.

E-mail: jakubsky@ujf.cas.cz

Nuclear Physics Institute of the ASCR, v. v. i.

Řž 130, 25068 Řž, Czech Republic 